\title{
The HEART (High Energy Astrophysical Radiative Transfer) code: modelling X-ray binaries and AGN
}

\author{
Katrine Rogers* \\ University of Southampton, UK \\ E-mail: ksreastro.soton.ac.uk \\ Omar Jamil \\ University of Southampton, UK \\ E-mail: o.jamileastro.soton.ac.uk
}

\section{Christian Kaiser}

University of Southampton, UK

E-mail: crk@phys.soton.ac.uk

\section{Ross Collins}

UK Astronomy Technology Centre, UK

E-mail: rsceroe.ac.uk

\begin{abstract}
We present a new, time dependent, three-dimensional code for modelling the inner regions of $\mathrm{X}$-ray binaries and AGN, where the photon distribution emitted by the accretion disc evolves simultaneously with the interacting electron distribution. The HEART (High Energy Astrophysical Radiative Transfer) code can model many different geometries including a spherical corona and an inhomogeneous corona. Inverse Compton scattering, bremsstrahlung, synchrotron radiation, Coulomb interactions, reprocessing of photons by the accretion disk, electron-positron annihilation and pair production are modelled. Electron distributions may be of power law form, thermal or a combination. We include some fits to X-ray spectral data and some results already obtained using the code.
\end{abstract}

VI Microquasar Workshop: Microquasars and Beyond September 18-22, 2006

Como, Italy

* Speaker. 


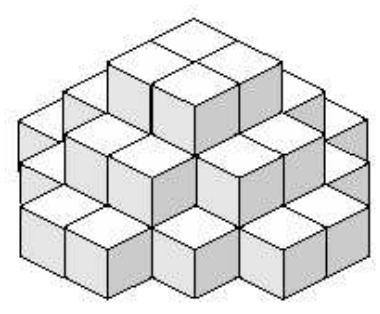

Figure 1: The cell structure of a semi-spherical corona with a width of 6 cells [1] .

\section{Introduction}

There is no satisfactory model of the geometry and physics of X-ray binaries and AGN explaining the spectra and variability we observe. We hope to make some progress on this issue by using HEART to model different geometries and more complete physical situations, including spectral and temporal variability.

\section{The code}

We use cubic cells to construct the geometry of the corona as practically any 3D shape can be approximated in this way. The corona is in the vicinity of a central black hole and (an optically thick, geometrically thin) accretion disc. Each cell knows its neighbours, allowing photons to transfer from one to the next.

Figure 1 displays half of a spherical corona, with 24 cells adjacent to the disc from which blackbody seed photons are injected into the corona. There are six possible photon distributions in each cell, one travelling in each of the six directions parallel to its sides. Each cell contains a hybrid electron distribution which can scatter the seed photons (and later, the scattered photons). While the photons are in a cell, any of the physical processes described may occur, and new electrons may be injected. In this way, the electron and photon distributions evolve simultaneously, and it is possible to reach equilibrium simultaneously in both the photon and the electron distributions. HEART can provide output at any point during a run, so it is possible to study the electron and photon distributions evolving as they reach equilibrium, or how they evolve if no equilibrium is reached.

Reprocessing is accounted for from the second timestep onwards (one timestep is the time taken for a photon to cross a cell). After scattering has occurred the photons reflected off the disc are injected together with the new blackbody seed photons. Finally when photons escape the corona, they are added to the output flux.

\section{Physical Processes}

\subsection{Radiative transfer}

We model synchrotron radiation, inverse Compton scattering and bremsstrahlung similarly. From [5] the radiative transfer equation for each process can be derived by calculating the emission 
and absorption coefficients:

$$
I_{v}\left(\tau_{v}\right)=S_{v}\left(1-e^{-\tau_{v}}\right),
$$

where $I_{v}$ is the intensity at frequency $v, \tau_{v}$ is the optical depth and $S_{v}=\frac{\varepsilon_{v}}{\kappa_{v}}$ where $\varepsilon_{v}$ and $\kappa_{v}$ are the emission coefficient and absorption coefficient respectively.

\subsection{Synchrotron Radiation and Bremsstrahlung}

The emissivity of synchrotron radiation is given in [5], but requires simplifying for efficient modelling. There is no satisfactory simplification covering all the modelled electron and photon energies, so we treat synchrotron radiation in 5 different cases, all of which can be simplified in different ways:

1. relativistic electrons $(\gamma \geq 2)$ in the limit $x \ll 1$,

2. relativistic electrons $(\gamma \geq 2)$ in the limit $x \gg 1$,

3. relativistic electrons $(\gamma \geq 2)$ for all other values of $x$,

4. non-relativistic electrons $(\gamma<2)$ where $v>v_{g}$,

5. non-relativistic electrons $(\gamma<2)$ where $v \leq v_{g}$.

where $\mathrm{x}$ is the emitted photon frequency divided by the critical frequency $\left(\frac{v}{v_{c}}\right)$ and $v_{g}$ is the gyro frequency.

For bremsstrahlung we only deal separately with the ultra relativistic $(\gamma \geq 2)$ and mildly relativistic cases in that we use different interaction cross sections. We use the treatments in [7], [3].

\subsection{Inverse Compton Scattering}

Inverse Compton scattering is treated slightly differently as it does not have an absorption coefficient. The radiative transfer equation is simplified based on the assumption that the corona is optically thin (within each individual cell) so that it only depends on the emissivity. We then consider three different cases to simplify the emissivity:

1. mildly relativistic electrons $(\gamma<10)$ interacting with high-energy photons $\left(k>10^{-2}\right)$,

2. mildly relativistic electrons $(\gamma<10)$ interacting with low energy photons $\left(k \leq 10^{-2}\right)$ and

3. relativistic electrons $(\gamma \geq 10)$, where $k=\gamma \frac{h v_{0}}{m_{e} c^{2}}$.

\subsection{Reprocessing}

Some photons are scattered in the corona and return to the disc where they may be absorbed or reflected. We use the treatment of [4] and [6] to model this reprocessing of photons. It adds an excess between $\sim 10$ and $100 \mathrm{keV}$.

\section{Electron Distributions}

We model hybrid electron distributions with thermal and power law components. However, any arbitrary distribution of electrons can be modelled. The distribution is homogeneous in each 

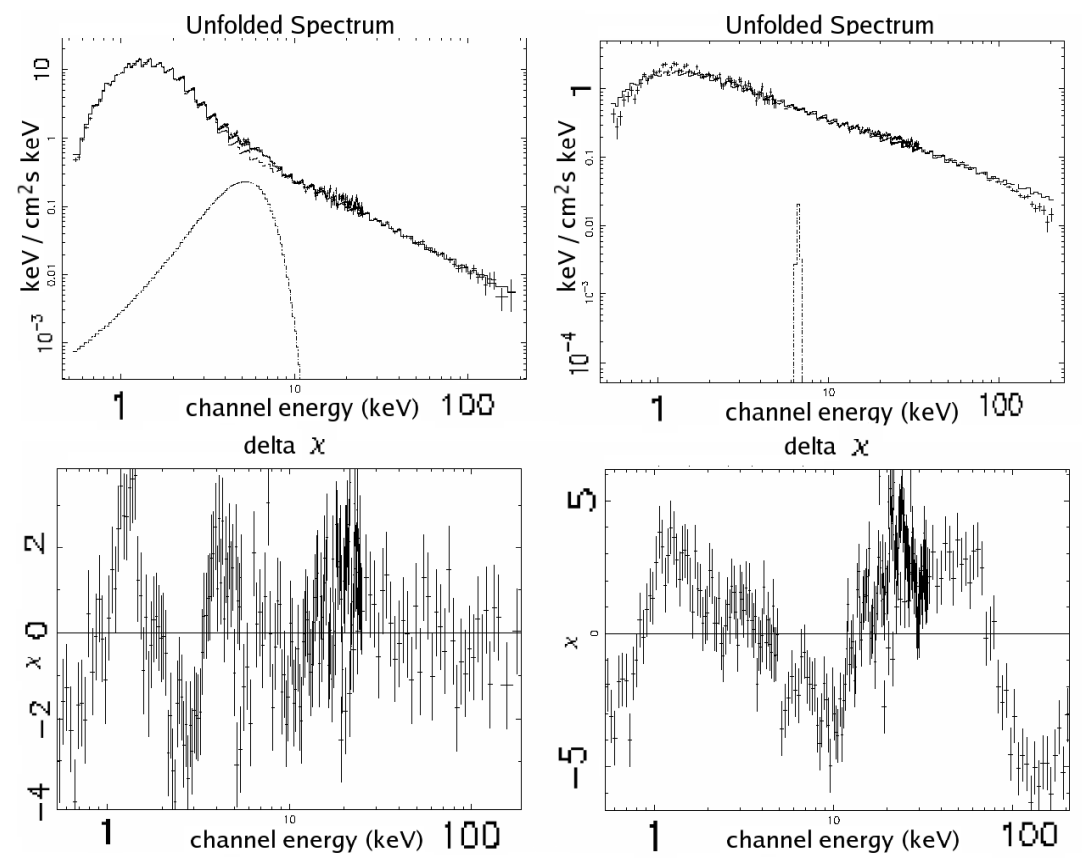

Figure 2: These are the best fits we have achieved so far with XSPEC. The soft state is on the left, with $\Delta \chi$ plotted beneath it, and the hard state with its $\Delta \chi$ plot is on the right.

cell, but may be inhomogeneous overall. Coulomb interactions thermalise the distribution and the kinetic equation takes account of energy losses due to interactions with photons. For more on electrons see Jamil et al., these proceedings.

\section{Results}

We are using HEART to model the soft and hard states of Cyg X-1 observed by BeppoSAX on June 22nd 1996 (in the low hard state) and on June 25th 1996 (in the high soft state). See [2] for details. We aim to show that HEART is capable of reproducing observations, and to explain the spectra with a more physical and complete model.

\subsection{Cyg X-1 in the high soft state and in the low hard state}

The best fits we have achieved in XSPEC so far are shown in figure 2. The fit parameters are:

\begin{tabular}{|l|l|l|}
\hline & High Soft State & Low Hard State \\
\hline $\mathrm{nH}$ & $4.82 \times 10^{21} \mathrm{~cm}^{-2}$ & $2.26 \times 10^{21} \mathrm{~cm}^{-2}$ \\
Fe line Energy & $4.75 \mathrm{keV}$ & $6.64 \mathrm{keV}$ \\
Fe line Sigma & $1.58 \mathrm{keV}$ & $0.10 \mathrm{keV}$ \\
Reduced chi-squared & 2.55 & 7.41 \\
Degrees of Freedom & 190 & 222 \\
\hline
\end{tabular}

All the physical processes described are used to model both states with HEART. In the high soft state, a purely powerlaw electron distribution with slope 1.65 and number density $2 \times 10^{20} \mathrm{~m}^{-3}$ 
$\mathrm{s}^{-1}$ is injected. The maximum temperature of the disc is $0.49 \mathrm{keV}$ and it extends from 3 to 100 Schwarzschild radii. No systematic errors have been accounted for. The low hard state has proved more challenging, and this is still a work in progress, but the best fit so far was achieved injecting a purely thermal electron distribution of number density $1.8 \times 10^{22} \mathrm{~m}^{-3} \mathrm{~s}^{-1}$ and temperature $650 \mathrm{keV}$. The maximum temperature of the disc is $0.001 \mathrm{keV}$ and it extends from 20 to 100 Schwarzschild radii. A systematic error of $1.5 \%$ has been accounted for.

\section{Future Work}

The above examples are simply illustrations of what HEART can do in the steady-state, homogeneous case. The strength of the code lies in the possibility of modelling inhomogeneous and time-dependent situations.

HEART is well suited to variability studies since we can easily study both spectral and temporal information as both the photon and electron distributions evolve in time. The geometry of the corona may also vary in time.

For example, we intend to simulate random, spontaneous bursts of high energy electrons, possibly caused by magnetic reconnection events. By using variability information to determine the timing of reconnection events, we aim to reproduce observed lightcurves and simultaneously study the effect on the spectrum.

HEART can be used similarly to investigate other possible physical explanations for variability in lightcurves, including long term variability.

We also intend to do similar analysis to that done for the low hard and high soft state above for the intermediate state. This should indicate which physical parameters reproduce the spectrum of the intermediate state as well as how these parameters evolve between states.

\section{References}

[1] Ross S. Collins, Modelling radiative transfer in high-energy astrophysical plasmas, $\mathrm{Ph} . \mathrm{D}$. thesis, School of Engineering Sciences, Faculty of Engineering, University of Southampton, 2004.

[2] R. Frontera, E. Palazzi, A. A. Zdziarski, F. Haardt, G. C. Perola, L. Chiappetti, G. Cusumano, D. Dal Fiume, S. Del Sordo, M. Orlandini, A. N. Parmar, L. Piro, A. Santangelo, A. Segreto, A. Treves, and M. Trifoglio, Broadband spectrum of cygnus $x-1$ in two spectral states with bepposax, ApJ 546, 1027-1037 (2001).

[3] R. J. Gould, Thermal bremsstrahlung from high-temperature plasmas, The Astrophysical Journal 238 , 1026-1033 (1980).

[4] Alan P. Lightman and Timothy R. White, Effects of cold matter in active galactic nuclei: A broad hump in the $x$-ray spectra, The Astrophysical Journal 335, 57-66 (1988).

[5] G. B. Rybicki and A. P. Lightman, Radiative processes in astrophysics, Wiley-Interscience, 1979.

[6] Timothy R. White, Alan P. Lightman, and Andrzej A. Zdziarski, Compton reflection of gamma rays by cold electrons, The Astrophysical Journal 331, 939-948 (1988).

[7] A. A. Zdziarski, P. S. Coppi, and D. Q. Lamb, Physical processes in photon-starved nonthermal pair plasmas, The Astrophysical Journal 357, 149-160 (1990). 